\title{
Spatial organization of nuclear pores in Xaenopus laevis oocytes
}

Linda Ravazzano ${ }^{1}$, Silvia Bonfanti ${ }^{1,2}$, Roberto Guerra ${ }^{1}$, Fabien Montel $^{3}$, Caterina A. M. La Porta ${ }^{4,5}$, Stefano Zapperi ${ }^{1,2^{*}}$

1 Center for Complexity and Biosystems, Department of Physics, University of Milan, Via Celoria 16, 20133 Milano, Italy

2 CNR - Consiglio Nazionale delle Ricerche, Istituto di Chimica della Materia Condensata e di Tecnologie per l'Energia, Via R. Cozzi 53, 20125 Milano, Italy

3 Université de Lyon, École Normale Supérieure de Lyon, Universit é Claude Bernard, CNRS, Laboratoire de Physique, Lyon 69342, France

4 Center for Complexity and Biosystems, Department of Environmental Science and Policy, University of Milan, via Celoria 26, 20133 Milano, Italy

5 CNR - Consiglio Nazionale delle Ricerche, Istituto di Biofisica, via Celoria 26, 20133

Milano, Italy

* stefano.zapperi@unimi.it

\begin{abstract}
The structure of nuclear pores has been the object of considerable investigation, but how nuclear pores are arranged on the nuclear surface is still less studied. Here, we analyze super-resolution images of the surface of Xenopus laevis oocytes nuclei during development and characterize the arrangement of nuclear pores using tools commonly used to study atomic structural and topological features of ordinary matter. To interpret the experimental results, we perform numerical simulations of octagonal clusters mimicking typical pore shapes and find structures that are in excellent agreement with experiments. The statistical features of the geometrical arrangement do not depend on the type of interaction between the pores, attractive or repulsive, but only on their octagonal geometry. We conclude that the observed arrangement of the pores is mainly dominated by their octagonal symmetry.
\end{abstract}

\section{Author summary}

The cell nucleus encloses the genetic materials of eukaryotic cells. Communication between inside and outside of the nucleus occurs through the nuclear pore complexes whose structures are composed by more than 30 different proteins arranged in an octagonal shape. Here, we study the spatial organization of nuclear pore complexes on the nuclear surface as revealed by super-resolution microscopy and compare the results with simulations of a set of octagonal particles. Our results suggest that the octagonal structure of the pores plays a central role on the way they are organized on the surface.

\section{Introduction}

In eukaryotic cells, genetic information is well protected inside the cell nucleus, divided by the outside cytoplasm by a membrane called nuclear envelope (NE), the most 
prominent nuclear scaffold. This segregation has the advantage of protecting the genome from sources of damage, but on the other side communications based on exchange of macromolecules, such as messenger RNAs (mRNA) or transcriptomic factors, are of vital importance during all the cell life cycle, in order to control protein synthesis and instruct gene expression $[1,2]$. The spatial architecture of the nucleus is crucial for the interaction between the genome and protein components of the nuclear complex and has a role in chromatin reorganization during cellular differentiation.

Nuclear pores, large protein assemblies inserted in the nuclear envelope, are responsible for selective nucleo-cytoplasmic transport allowing the free diffusion of ions and small molecules and acting as selective gates for import and export of macromolecules such as proteins and mRNAs [3]. Furthermore, nuclear pores are also involved in the organization of the genome and contribute to gene regulation through physical interactions with chromatin [4] Since the discovery of nuclear pores in the 1950s, considerable effort has been devoted to understanding their peculiar structure via Electron Microscopy (EM) and more recently via Cryo-Electron Tomography (cryo-ET) [5]. Nuclear pores appear as modular assemblies of discrete constituents arranged with octagonal symmetry around a central axis [6]. Later on, those discrete elements have been identifyed with multiple copies of about 34 protein subunits (nucleoporins), remarkably conserved throughout eukaryotes, showing similar features in algae, yeast, vertebrates such as Xaenopus Laevis up to human. The characteristic shape of a nuclear pore consists of two superimposed rings of nucleoporins, with eightfold symmetry, one on the outer face of the nuclear membrane and one on the inner face; eight extended filaments depart from each ring. In contrast, the center of the pore, which forms the permeability barrier, is filled with disordered filaments of phenylanine-glycine repeats(FG) $[1,5]$.

Despite great progress in understanding the structure of a single nuclear pore, little is known about how nuclear pores are distributed across the surface of the nuclear membrane (in an average human cell there are approximately 2000-3000 nuclear pores) and whether and how they might interact among each other. Interactions are likely modulated by the the nuclear lamina, a filamentous protein network underlying the nuclear envelope, but how the interaction occurs is still unclear. Interestingly, earlier studies revealed that highly proliferative cells such as embryos or tumors have an high density of nuclear pores on the nuclear membrane, while terminal differentiated cells have fewer, suggesting a link between number and distribution of pores and cell activity [7]. This link has been explored in another early study focused on the changes in distribution of nuclear pores during spermatogenesis, following the evolution from spermatocytes to early spermatids. In particular, a clear change in nuclear pore spatial organization, from aggregation with hexagonal packing in pore rich areas coexisting with large pore-free areas in spermatocytes to a random distribution of pores in early spermatids has been observed [8].A further step in our understanding of the role of nuclear pore organization came with the observations of large pore-free islands in HeLa S3 human cells. These islands disperse with cell-cycle progression and reveal the importance of lamin $\mathrm{A} / \mathrm{C}$ in regulating the pore distribution [9].

In a recent paper, Sellés et al. performed super-resolution microscopy on Xaenopus laevis oocytes observing the variation of nuclear pore distribution on the nuclear membrane during oocyte development [10]. In this paper, we investigated the way nuclear pores are distributed across the nuclear membrane during oocyte development in Xaenopus laevis, analyzing available experimental results [10] with tools typical of the physics of soft materials, such as the calculation of the radial distribution function (RDF), local order parameters and Voronoi tessellation. We then considered the hypothesis of an effective interaction acting among the nuclear pores, in order to study if this can explain the spatial distribution of pores on the nuclear membrane. To this 
end, we built a potential with octagonal symmetry that models the interaction between nuclear pores and performed extensive numerical simulations at different pore density. The simulated configurations we obtained represent a first attempt of studying in silico the nuclear pore complex globally, to unveil peculiarities and characteristics of spatial distribution of nuclear pores.

\section{Materials and methods}

\section{Experimental images}

We analyze the experimental images of nuclear pores of Xenopus laevis oocyte by Selles et al. [10]. According to the stage of development of the oocyte [11] we identify three groups of images: from the early Stage II, to an intermediate Stage IV, to a later Stage VI. A different number of samples was taken at each stage, specifically, 6 samples for Stage II, and 11 samples for both Stage IV and Stage VI. The images are $2560 \times 2560$ pixels (px) wide, with $1 \mathrm{px}$ corresponding to $0.01 \mu \mathrm{m}$.

\section{Tracking of Nuclear Pores}

We analyze the trajectory of the nuclear pores with Trackpy v0.4.2, a Python package for particle tracking in 2D, 3D, and higher dimensions [12]. In particular, we use the function trackpy.locate routine to first discriminate the nuclear pores. The working principle is the following: i) preprocess the image performing a bandpass filter (i.e. performing a convolution with a Gaussian to remove short-wavelength noise and subtracting out long-wavelength variations by subtracting a running average, in order to retains features of intermediate scale), ii) applying a threshold over the color channels, and iii) locating all the peaks of brightness, each referring to the position of a pore. $[12,13]$. The parameters used for the tracking are diameter $=9 \mathrm{px}$, minmass (the minimum integrated brightness, working as a threshold value) value instead is chosen according to the samples. If minmass $=0$ so no threshold is used in the high density samples of Stage II, higher values of this parameter are necessary to correctly detect pores in the more noisy experimental images of Stage IV and VI.

From this tracking procedure we extracted the 2D coordinates of the nuclear pores, on which our statistical analysis is based. The tracking procedure also allowed us to determine the density in terms of number of pores for nuclear envelope surface unit of the experimental samples: $34.9 \pm 2.3 \mathrm{NPC} / \mu \mathrm{m}^{2}$ for Stage II, $25.6 \pm 2.3 \mathrm{NPC} / \mu \mathrm{m}^{2}$ for Stage IV, $20.5 \pm 1.7 \mathrm{NPC} / \mu \mathrm{m}^{2}$ for Stage VI (errors represent the standard deviation computed on the ensemble of samples for each developmental Stage). Those density values are a bit underestimated respect to the ones computed by Sellès et al. [10], but this is due to different techniques used for tracking procedure and to an intrinsic uncertainty connected with the experimental measures performed by optical super-resolution microscopy that introduce some arbitrarily in identifying as pores some spot 'fragmented' due to small microscope displacement, so affecting the overall counting.

\section{Numerical Simulations}

To model the interaction among nuclear pores, we consider their peculiar octagonal shape, as observed in early experimental studies [6], [14] and subsequently confirmed by structural studies on nucleoporins - a family of proteins that are the main components of the nuclear pore complex in eukaryotic cells - and by recent advancements in experimental techniques such as cross-linking mass spectrometry and cryo-electron tomography [5]. However, while the structure of a single nuclear pore is nowadays well 
understood, the way these pores interact each other on the surface of the cellular nucleus still remains to be investigated. In the following paragraph we elucidate the details of our simulation model which consist of a simple potential describing the pore-pore interaction.

\section{Model Potential for Nuclear Pores}

To take into account the composite structure of each nuclear pore and its overall octagonal shape, we build a coarse-grain model of the pore, consisting of a central particle surrounded by eight particles located at the vertices of a regular octagon, of circumradius $R$. For simplicity, the so-modeled pore is treated as an undeformable rigid object. Following reference experimental data [15], [10], in all the simulations we have set $R=67.5 \mathrm{~nm}$. The overall interaction potential acting among the particles of two neighboring pores is made up of three terms, each consisting in a Lennard-Jones (LJ) potential,

$$
\begin{array}{r}
V\left(r_{i j}\right)=4 \cdot \varepsilon \cdot\left(\left(\frac{\sigma}{r_{i j}}\right)^{12}-\left(\frac{\sigma}{r_{i j}}\right)^{6}\right), \\
r_{i j}<r_{c u t}
\end{array}
$$

with $r_{i j}=\left|\mathbf{r}_{i}-\mathbf{r}_{j}\right|$ is the distance between particle $i$ and particle $j$, and $\varepsilon, \sigma$, and $r_{c u t}$ parameters depend on the interaction term:

- center-center interaction - the central particle of a pore interacts repulsively with the central particle of a neighboring pore. For this term we have set $\varepsilon_{c c}=0.01 \mathrm{pg} \cdot \mu \mathrm{m}^{2} / \mu \mathrm{s}^{2}, \sigma_{c c}=0.12 \mu \mathrm{m}$, and the cutoff distance is set $r_{c c}^{c u t}=2^{1 / 6} \sigma_{c c}$ so to make the interaction purely repulsive. This term is necessary to prevent unphysical configurations such as the case of overlapping pores that were otherwise seldom encountered.

- center-vertex interaction - the central particle of a pore repulsively interacts with the vertex particle of a neighboring pore. Again this is introduced to avoid pores overlap and compenetration. LJ parameters for this term are $\varepsilon_{c v}=0.01$ pg. $\mu \mathrm{m}^{2} / \mu \mathrm{s}^{2}, \sigma_{c v}=0.08 \mu \mathrm{m}, r_{c v}^{c u t}=2^{1 / 6} \sigma_{c v}$.

- vertex-vertex interaction - the vertex particle of a pore interacts with the vertex particle of a neighboring pore. We first considered full LJ interaction (long range attractive, short range repulsive), but the purely repulsive case was also investigated (see Supplementary S1 Fig). For the LJ interaction we have set $\varepsilon_{v v}=5 \cdot 10^{-4} \mathrm{pg} \cdot \mu \mathrm{m}^{2} / \mu \mathrm{s}^{2}, \sigma_{v v}=0.02 \mu \mathrm{m}$, and the cutoff distance is set to $r_{v v}^{c u t}=2.5 \sigma_{v v}$ so to include the attractive part. For the purely repulsive case we have set $r_{v v}^{c u t}=2{ }^{1 / 6} \sigma_{v v}$. By construction, since the central particles are surrounded by vertex particles, in our model the overall pore-pore interaction is mostly driven by the present term.

In all the cases the LJ potentials are shifted so to zero out at the cutoff distance, in order to avoid any energy discontinuity. Fig. 1 reports the total interaction energy of an octagonal pore (centered at the origin) with one corner particle of a second pore, as a function of the position of the latter. The resulting potential energy surface (PES) shows a strongly repulsive core region (in blue) and trapping regions (in red) concentrated at the proximity of the eight corner sites. 


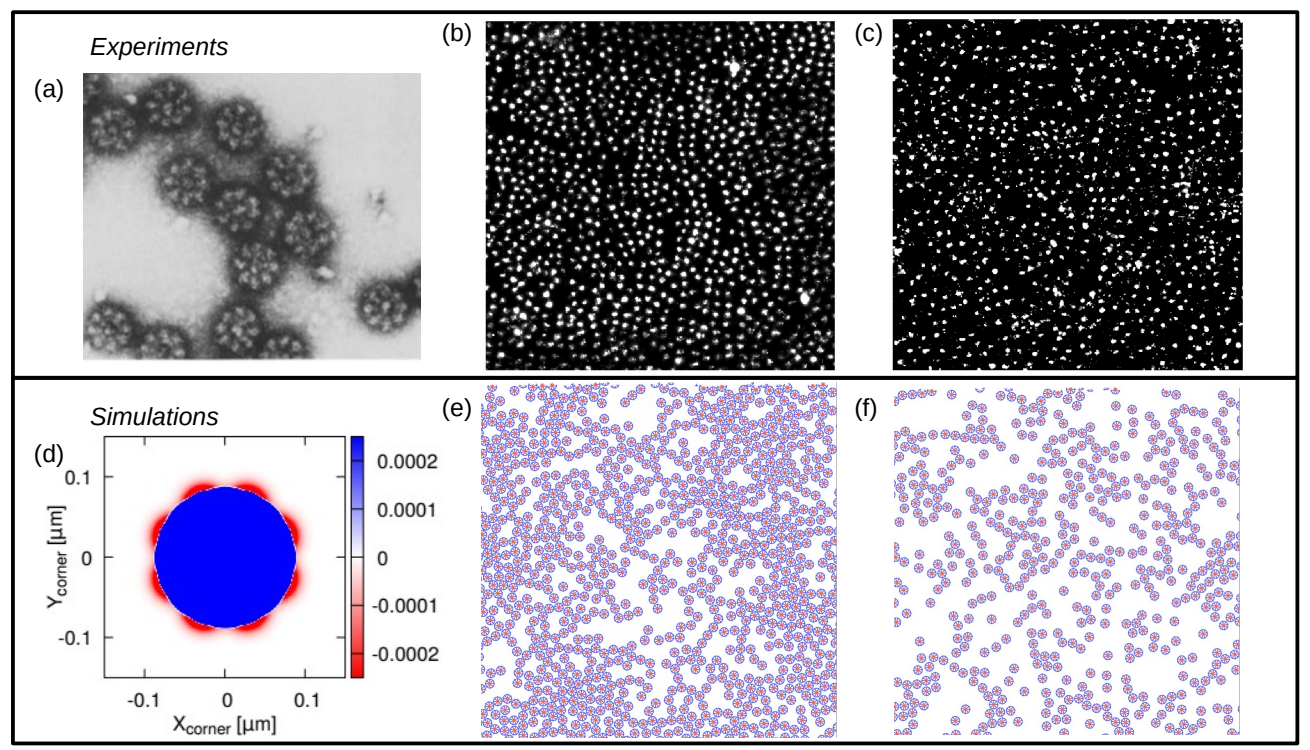

Fig 1. Visual comparison of real nuclear pores coming from experimental images and simulated nuclear pores (a) Detached pore complexes released onto the microscope grid from a nuclear envelope immersed in low salt medium containing $0.1 \%$ Triton $X-100$, from which the octagonal shape is clearly visible; (b),(c) portions of experimental images of nuclear pores in Xenopus laevis oocyte at different developmental stages, Stage II (b) and Stage VI (c), respectively, obtained using super-resolution microscopy. Panels (a) is an adaptations from Unwin et al. [6], panels (b) and (c) are adaptations from Sellés et al. [10]; (d) potential energy surface obtained from the modeled interaction between a pore and a corner of a neighboring pore: blu area marks a strongly repulsive region, while red areas mark trapping centers; (e) and (f) two configurations of nuclear pores obtained from simulations, for comparison with experimental data. The density is $36 \mathrm{NPC} / \mu \mathrm{m}^{2}$ for (e) and $20 \mathrm{NPC} / \mu \mathrm{m}^{2}$ for (f).

\section{Obtaining Configurations of Simulated Nuclear Pores}

- Simulations of nuclear pore assemblies were performed using LAMMPS [16] with a timestep $\Delta t=10^{-5} \mu \mathrm{s}$. The starting point are initial configurations of 1000

randomly-positioned octagonal pores confined in a periodic square box side $L=40 \mu \mathrm{m}$. To mimic the different experimental densities of nuclear pores observed during the oocyte development, the random configurations undergo alternatively $10^{5}$ steps of box compression at a constant temperature $T=\frac{2}{3} \frac{\epsilon_{c o r n}}{k_{B}}$ followed by a $2 \cdot 10^{5}$ steps annealing from temperature $T=\frac{\epsilon_{\text {corn }}}{k_{B}}$ to $T=\frac{2}{3} \frac{\epsilon_{\text {corn }}}{k_{B}}$, so to allow thermally-assisted

rearrangements. Such procedure is iterated until the required density is obtained, and a final energy minimization at $T=0 \mathrm{~K}$ in $2 \cdot 10^{6}$ steps is then performed. Following the above protocol we obtained configurations with a density of $20,26,36,46$, and 53 $\mathrm{NPC} / \mu \mathrm{m}^{2}$. For each density value we obtained 10 different realizations starting from different random initial positions, in order to permit proper statistical averaging.

\section{Statistical Analysis of Nuclear Pores Structure}

To provide a statistical comparison of simulations with reference experimental data we have applied three different post-processing techniques over the simulated pore assemblies and to their experimental counterparts. 


\section{Radial Distribution Function}

- To gain insight on the local structure of the nuclear pore complex on the nuclear membrane, we made use of the Radial Distribution Function (RDF) [17]:

$$
g(r)=\frac{L^{2}}{2 \pi r N^{2}} \sum_{i=1}^{N} \sum_{\substack{j=1 \\ j \neq i}}^{N}\left\langle\delta\left(r-r_{i j}\right)\right\rangle
$$

where $N$ is the number of particles in the system, $L$ is the system size and $r_{i j}$ is the distance between particle $i$ and $j$. The RDF has proven a key role in the theory of monatomic liquids, and has already been used to characterize amorphous colloidal solids [18] and to study glass transitions in Lennard-Jones systems [19].

\section{Hexatic Order Parameter}

- To better characterize the geometrical properties of the structure formed by the nuclear pores, we computed for each particle the $\mathrm{n}$-fold local orientational order parameter:

$$
\psi_{n}\left(r_{i j}\right)=\frac{1}{n n n} \sum_{j=1}^{n n n} e^{i n \theta\left(r_{i j}\right)}
$$

where $n n n$ is the number of nearest neighbors of particle $i, \theta\left(r_{i j}\right)$ is the angle formed by the $x$ axis and the vector $\overrightarrow{r_{i j}}$ connecting particles $i$ and $j$. Since ordered regions marked by the presence of square lattice and triangular lattice have been observed in experimental nuclear pore complexes of Xenopus laevis at different developmental stages [10], we focus our analysis on the order parameters with $n=6$ for which $\left|\psi_{6}\right|=1$ for particles belonging to a perfect hexagonal structure, and with $n=4$, for which $\left|\psi_{4}\right|=1$ for particles belonging to a perfect square lattice. The determination of nearest neighbor particles is done using a cutoff distance $\sigma_{c u t}=0.16 \mu \mathrm{m}$ for the simulations and $\sigma_{c u t}=0.20 \mu \mathrm{m}$ for the experimental images. Those values have been chosen looking at typical inter-particle distances in different samples of experimental images and simulated configurations respectively. All the isolated particles $(n n n<2)$ have been marked by zeroing their $\psi_{n}$ value.

\section{Voronoi Tessellation}

- Around the center of each pore we have built a Voronoi cell, an area of space containing all points that are closer to one pore than to any other particle. For the experimental images, pore centers coordinates are obtained from the tracking procedure and used as 'seeds' for the Voronoi tesselation. For the simulations, instead, only the central particle of each octagon has been considered in the Voronoi analysis. By construction, each Voronoi cell has polygonal shape, with a number of sides that corresponds to the number of neighbours. To compute the Voronoi tesselation we used the Python library Freud [20], that allows to account for periodic boundary conditions. By this method we managed to extract for each particle the number of neighbors (particles are considered neighbors if they share an edge in the Voronoi diagram) and the size of each associated Voronoi cell. 


\section{Results}

\section{Global structure of NPC}

Starting from the observation of the Radial Distribution function of the experimental samples, we noticed that at high density (so early stage of development of the oocyte), $g(r)$ shows a liquid-like shape, with two peaks clearly visible as in Fig 2a. In fact in monoatomic liquids $g(r)$ vanishes as $r \rightarrow 0$ as a consequence of the strongly repulsive forces that act at small particles separations, shows a pattern of peaks representing "shells" of neighbours around the reference particle and then tends to unity at large $\mathrm{r}$ underlying the loose of ordered structure and random disposition of particles (long range disorder) [17]. As the density decreases during oocyte development, the second peak of the $\mathrm{g}(\mathrm{r})$ tends to disappear and the first one flattens, so turning towards a gas-like behavior in which also the short range order is lost soon. Since previous analysis on the experimental images suggested a significantly presence of square lattice domains of nuclear pores at low density (Stage II) [10], we looked for a peak situated at $x_{s q}=\sqrt{2} x_{1}$ where $x_{1}$ represent the coordinate of the first peak of the $\mathrm{g}(\mathrm{r})$ function. What we noticed is that a peak in those peculiar position is not present, although $x_{s q}$ falls on the tail of the flat first peak, meaning that some regions with square lattice could be present inside the amorphous liquid, but do not influence significantly the local structure of the Nuclear Pore Complex. We then analyzed the $g(r)$ obtained from our simulations with octagonal attractive potential, and we noticed again a typical liquid-like shape, with two peaks clearly visible at different densities (see Fig 2) and with peaks positions that tends to be integer multiples of the first peak position. The most important information gained comparing the $\mathrm{g}(\mathrm{r})$ of experiments and simulations is that the latter could describe quite well the location of the peaks of the experimental samples, meaning that our model is able to catch important features of the effective interaction among nuclear pores on the nuclear membrane. In fact the peak positions strictly reflects the interactions that hold among particles and are a signature of each material and its peculiar properties, as showed in previous studies on noble gases or water [21], [22], [23], [24]. In the end from this analysis we exclude the presence of significative regular regions, as already mentioned in the case of square lattice, and also for what concern triangular lattice. About that what we noticed is that $x_{t r i}=\sqrt{3} x_{1}$ falls on the growing part of the second peak for the high density configurations, representing a first clue of the presence of some hexagonal structures at high density.

\section{Orientational Order}

We calculated the local order parameters $\psi_{6}$ and $\psi_{4}$ as defined in (4) for all the experimental samples and the configurations obtained from our simulations. The cutoff to consider a particle a neighbor is $\sigma_{c u t}=0.20 \mu \mathrm{m}$ for the experiments and $\sigma_{c u t}=0.15$ $\mu \mathrm{m}$ for the simulations; those values have been chosen extracting from the images the typical inter-particles distances. In Fig3a and Fig3c the particles colored in red are the ones with $\psi_{4} \simeq 1$, so the ones belonging to square lattice structures. It can be observed that only few particles belong to local ordered structures. In Fig3b and Fig3b instead, the particles colored in red are the ones with $\psi_{6} \simeq 1$, so the ones belonging to triangular lattice structures. In those samples, having an higher value of nuclear pore density, more particles belong to a regular structure and an hexagonal order appears in some regions. To better investigate this behavior we computed the distribution of the local order parameters $P\left(\psi_{6}\right)$ and $P\left(\psi_{4}\right)$ averaged on all the samples for each value of the density and we plotted them in Fig4. The first important feature that can be noticed from the orientational order distributions of the experimental samples (Fig 4a and Fig $4 \mathrm{~b}$ ) is that those distribution are all unimodal, so excluding the hypotesys of two 

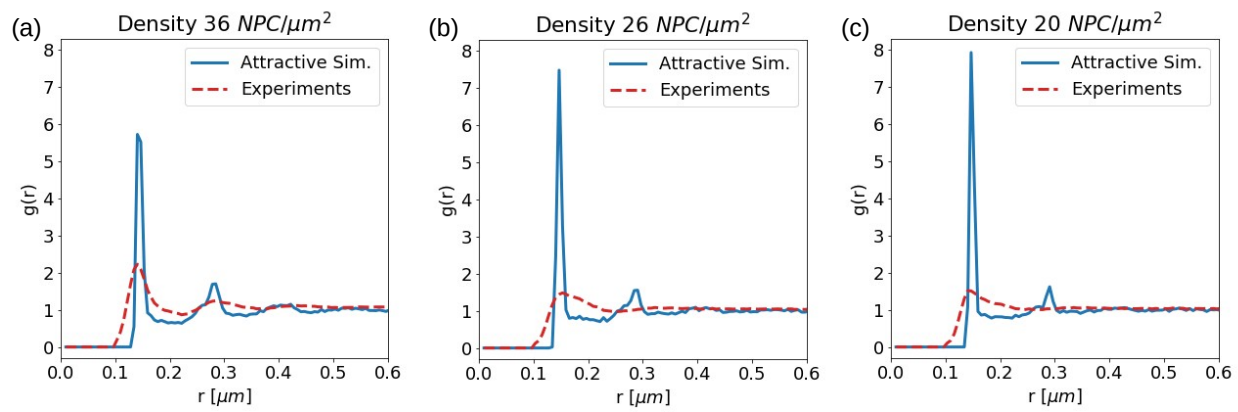

Fig 2. Radial Distribution Function of nuclear pores The Radial Distribution function $\mathrm{g}(\mathrm{r})$ is represented for three different values of density of the nuclear pores. In blue the function plotted for our simulations with attractive octagon potential, averaged on ten configuration each. In dashed red the function averaged on the experimental samples for Stage II - (a), Stage IV - (b), Stage VI - (c).

different cohexisting phases as suggested in previous analysis [10]. The other information that can be extracted is that the peak of the distribution is shifted toward higher values increasing the density both in the case of $\psi_{6}$ and $\psi_{4}$, but at high density (Stage II) the $\psi_{4}$ distribution peak reaches lower values respect to the $\psi_{6}$ one, suggesting a preference for hexagonal structures in the dense limit. Those characteristics can be observed also in the results obtained from our simulations in Fig4c and Fig4d. For a better comparison we plotted in Fig4e the value of $\left|\psi_{6}\right|$ and $\left|\psi_{4}\right|$ averaged on particles and samples for our simulations (dashed lines) and the results obtained from the experimental samples (points). Looking at the dashed lines, we observe that both $\left|\psi_{6}\right|$ and $\left|\psi_{4}\right|$ slowly increase with density showing the same values until a density of $36 \mathrm{NPC} / \mu \mathrm{m}^{2}$ in which a bifurcation occurs. From that value of density $\left|\psi_{4}\right|$ seems to reach a plateu while $\left|\psi_{6}\right|$ continues to increase, so favouring the hexagonal order at high density. It has to be noticed that, in the explored density range, the $\left|\psi_{6}\right|$ does not reach a value compatible with crystalline structure. The experimental points do not fall exactly on the theoretical curves predicted by the simulations, and this can be partially explained with the uncertainties connected with the experimental observations of the nuclear pores, that affect the density evaluation. Despite that, noticeably the experimental points show the same trend of the simulation, with an initial overlapping of $\left|\psi_{6}\right|$ and $\left|\psi_{4}\right|$ and a further bifurcation at higher density.

\section{Properties of Voronoi cells}

We performed a Voronoi tesselation on the nuclear pores centers extracted both from the experimental images and from the configurations obtained via our simulations. In Fig5a and Fig5d two examples of this tesselations are shown: in those high density samples the similarity between experiments and simulations can be appreciated. Analyzing the number of neighbors of each Voronoi cell we plotted the histograms in Fig5b and Fig5e. It can be seen that the height of bar for $N=4$ decreases increasing 
bioRxiv preprint doi: https://doi.org/10.1101/2021.09.01.458492; this version posted September 15, 2021. The copyright holder for this preprint (which was not certified by peer review) is the author/funder, who has granted bioRxiv a license to display the preprint in perpetuity. It is made available under aCC-BY 4.0 International license.

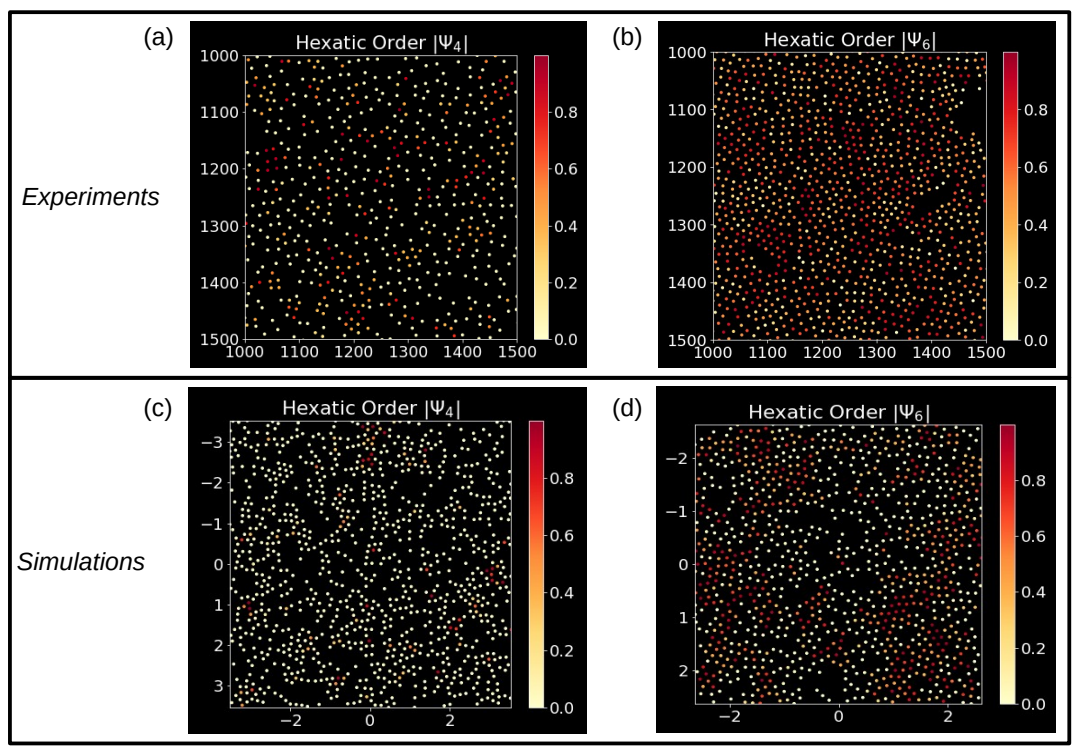

Fig 3. Local order parameters colored maps In (a) a zoomed region of an experimental sample at Stage VI is represented, in (b) a zoomed region of an experimental sample at Stage II. For comparison a simulation box at density 20 $\mathrm{NPC} / \mu \mathrm{m}^{2}-(\mathrm{c})$ and density $36 \mathrm{NPC} / \mu \mathrm{m}^{2}$ are shown.
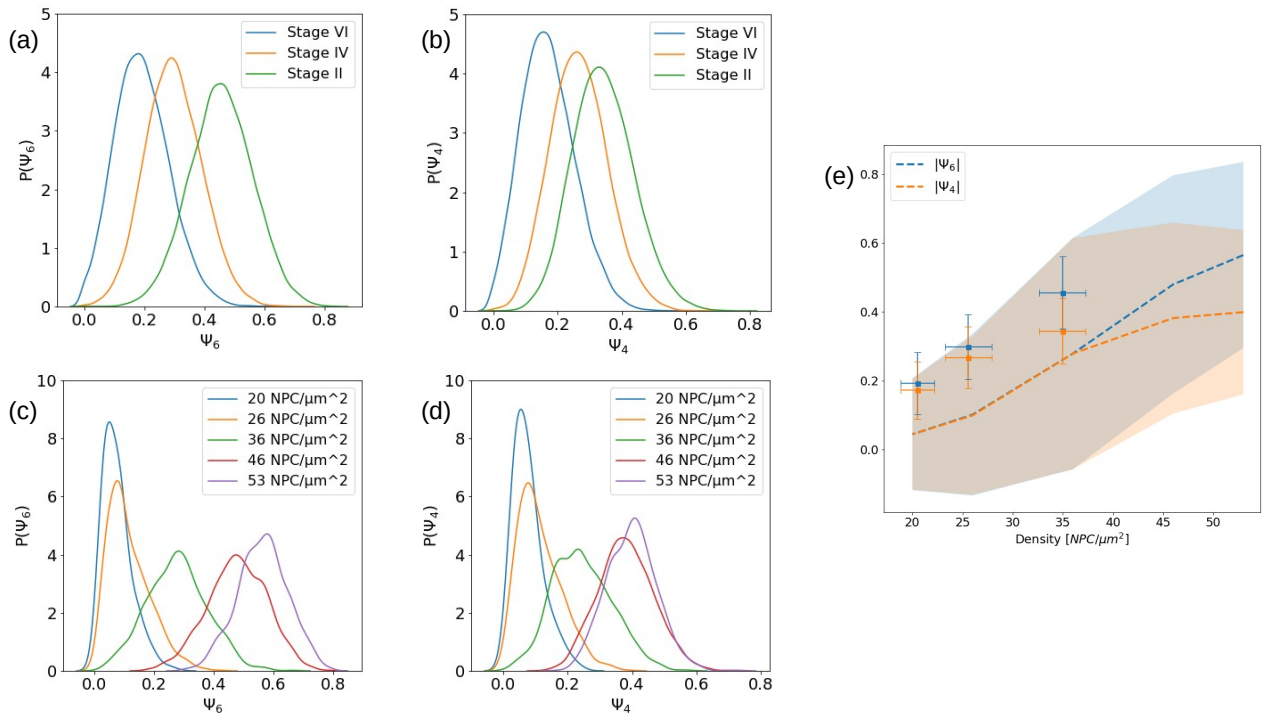

Fig 4. Distributions of local order parameters The distribution of the local order parameters averaged on different experimental samples (a) and (b) and different configurations obtained from the simulations (c) and (d) are plotted for each density value considered. In (e) from the above mentioned distributions we computed the $\left|\psi_{6}\right|$ and $\left|\psi_{4}\right|$ averaging also among particles. The dashed lines represent value expected from the simulations, the shadows representing a standard deviation. The points represent the value computed from the experimental samples, again the errorbars on y stands for a standard deviation from the averaged computed value of the local order parameter, while the errorbars on $\mathrm{x}$ represent the error on density determination as described in Section 0.1.1 
density while the bar for $N=6$ shows the opposite trend, increasing with increasing density. So the Voronoi analysis on one side confirms what already observed using the local order parameter, that the hexagonal configuration is favoured at high densities. On the other side seems to partially confirm what observed experimentally, so the presence of some square structure at low density. This point is yet controversial, because from the histogram we see that also the $N=5$ bar show a behavior similar to the $N=4$ one, probably because the number of 'defects' of the Voronoi tesselation decreases with increasing density. Finally we computed the distributions of the Voronoi cells size and plotted them in Fig5c and Fig5e. Again a good accord between the experiments and our model can be observed, with a narrow distribution at high density, almost centered on the nuclear pore volume, that shifts its peak toward higher volume values and becomes flatter decreasing the density.

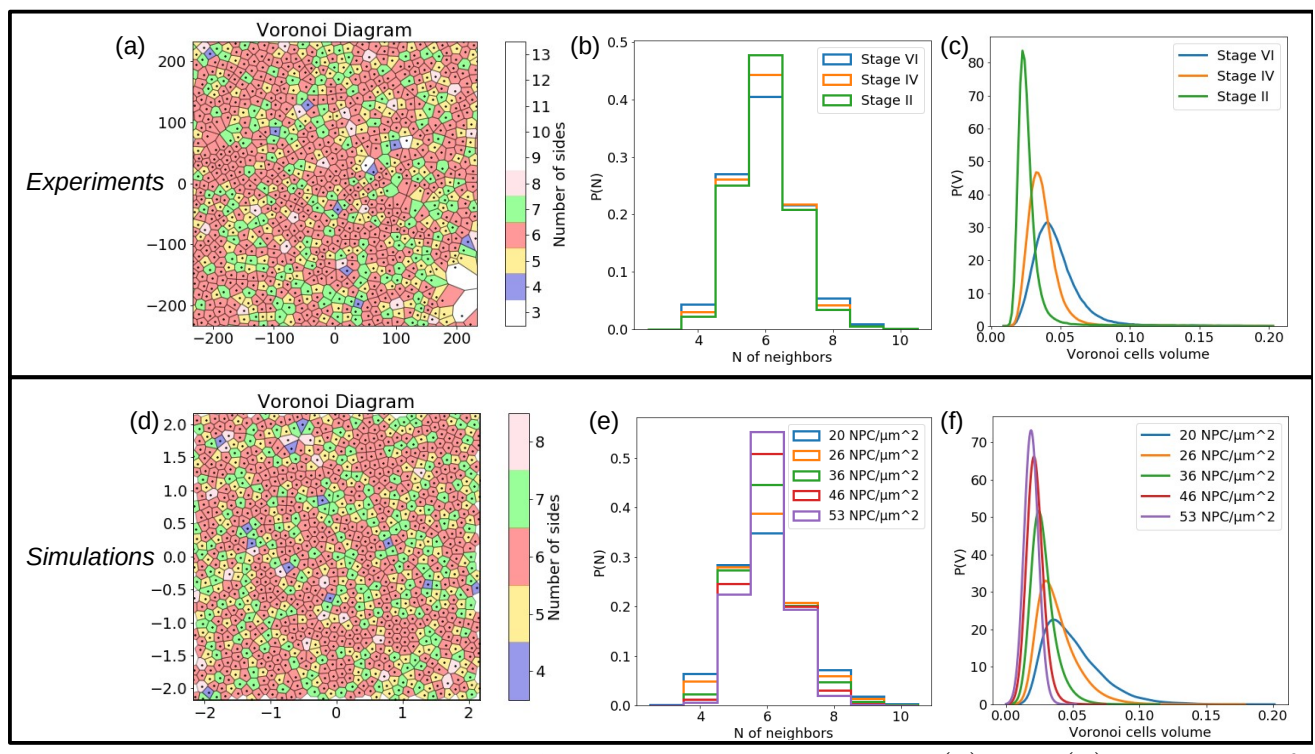

Fig 5. Voronoi tesselation applied to nuclear pores In (a) and (d) examples of Voronoi tesselation are given for an experimental samples at Stage II and for a simulation with density $46 \mathrm{NPC} / \mu \mathrm{m}^{2}$ respectively. In (b) and (e) the histogram of the number of nearest neighbors computed counting the sides of the Voronoi cells are represented. In (c) and (d) we plotted the distributions of the volume, expressed in $\mu \mathrm{m}^{2}$, of the Voronoi cells at different densities.

\section{Discussion}

Having calculated the radial distribution functions of nuclear pores on nuclear membrane of Xaenopus laevis oocytes allowed us to identify an amorphous, liquid-like structure in which the long range order is lost soon (just two peaks are clearly visible) at early stage of oocyte development, where the density of NPCs is high. Instead, proceeding during the development of the oocyte, the density of nuclear pores decreases and the radial distribution function shows a more dilute, gas-like behavior. Under a biological point of view, the early stages of oocyte development are associated with high transcriptional activity, since the oocyte needs to build up a huge store of gene products such as mRNAs, tRNAs and proteins to correctly perform its future role after fertilization. Once that the maternal mRNAs needed are transcribed, the transcriptional activity becomes low in later stage of oocyte development [25]. Those 
changes in transcriptional activity could be connected with the variations of the NPCs spatial distribution during oocyte development, especially for what concerns changes in density. Even more interesting is to look at the locations of the peaks in the radial distribution functions we computed. In fact, they give direct information on the geometrical arrangement of particles inside a material and besides that they allow to extrapolate as indirect information what kind of interaction holds among the particles, since peak locations and their relative distances represent a signature for each material [26]. In this direction, a first attempt to investigate the spatial distribution of nuclear pores goes back to the '70s, when the positions of NPCs on the surface of rat kidney nuclei was observed and distances among them measured. Already at those times, some regularities were found in the distribution of pore-pore distances measured in the samples, suggesting a non random spatial distribution and some peaks corresponding to hexagonal structures, even if the statistic was too poor to reach further conclusions [27]. More recently Sellés et al. looked at the angular distribution between first neighbors for each nuclear pore, revealing no preferential angles for Stage II and IV Xaenopus laevis oocytes (high density) and two distinct peaks at $90^{\circ}$ and $180^{\circ}$ for later Stage VI, underlying the presence of square lattice regions at low density [10]. In our study we did not focused on single nearest neighbor but on shells on neighbours using the tecnique of the $\mathrm{g}(\mathrm{r})$, as usually done in the contest of liquids and amorphous solids. From the radial distribution function we did not observe peaks in correspondence of peculiar geometrical structures, meaning that also if some regular regions are present, they are quite rare and do not statistically influence the overall NPC spatial

distribution. On the other side, the very interesting result we reached with our study is that our eight-fold potential used to model NPCs in our simulations, is able to predict very well the experimental peak location. The position of the first peak obtained from our simulations, is not only in excellent agreement with the experimental samples by Sellés et al. [10], but also with previous studies [27], [8]. This is absolutely non trivial, since we built our computer simulations on the assumption of an effective interaction among NPCs, which peculiar feature is the eight-fold simmetry. In fact, looking at the supplementary S1 Fig, it can be observed that a spherical potential describing the single nuclear pore in its interaction with the surrounding ones, fails in correctly identifying the peak location in the $\mathrm{g}(\mathrm{r})$, suggesting that the octagonal shape of the NPCs plays a role in determining their spatial distribution. But even more important is the fact that our very simple model, based on an effective potential can tell us something about interaction among nuclear pores, that is in reality much more complicated, allowing to produce in silico possible configurations controlling only few key parameters. In reality, the spatial arrangement of NPCs will be mediated by the lamin scaffold through complex interactions that are not easy to model explicitly.

Coming back to the search of local regularities in the spatial distribution of NPCs, we interestingly observed from the study of the local order parameters that at high density the pores tend to arrange following the triangular lattice, forming hexagonal structures. In fact, even if the $\mathrm{g}(\mathrm{r})$ does not report explicit peaks in correspondence of triangular lattice, from the $\Psi_{6}$ colored map in Fig. 3 and from the Voronoi tesselation in Fig. 5 it is possible to see that at high density islands of hexagonal packed pores appear. Noticeably this kind of behavior has been reported in previous experimental observations. During apoptosis the distribution of nuclear pores on the cell nucleus strongly changes, bringing the NPCs to be highly concentrated in small regions of the nuclear envelope (on mouse cell nuclei) and leaving the rest of the surface pore-free. Those clusters of pores showed an hexagonal packing and were supposed to be correlated with diffuse chromatine areas [28]. Occasional areas of very regular hexagonal packing of nuclear pores have been also observed to emerge during the development of male germ cells, in rodent spermatocytes [8]. Those facts open interesting questions on 
how the geometrical disposition of the pores in some areas, or even more simply, their density, are influenced by the underlying nuclear activity and on what are the biological causes responsible for the effective interaction among NPCs.

\section{Conclusion}

In the present paper, we analyzed the spatial organization of nuclear pore on the nuclear surface as a function of the development stage in Xaenopus laevis oocytes. To this end, we use statistical indicators conventionally used to described phase-transitions in two dimensional liquids. Our analysis indicate of experimental images combined with molecular dynamics simulations highlight tthe relevance of the octagonal symmetry of the pore in determining spatial patterns.

\section{Supporting information}

\section{S1 Fig.}
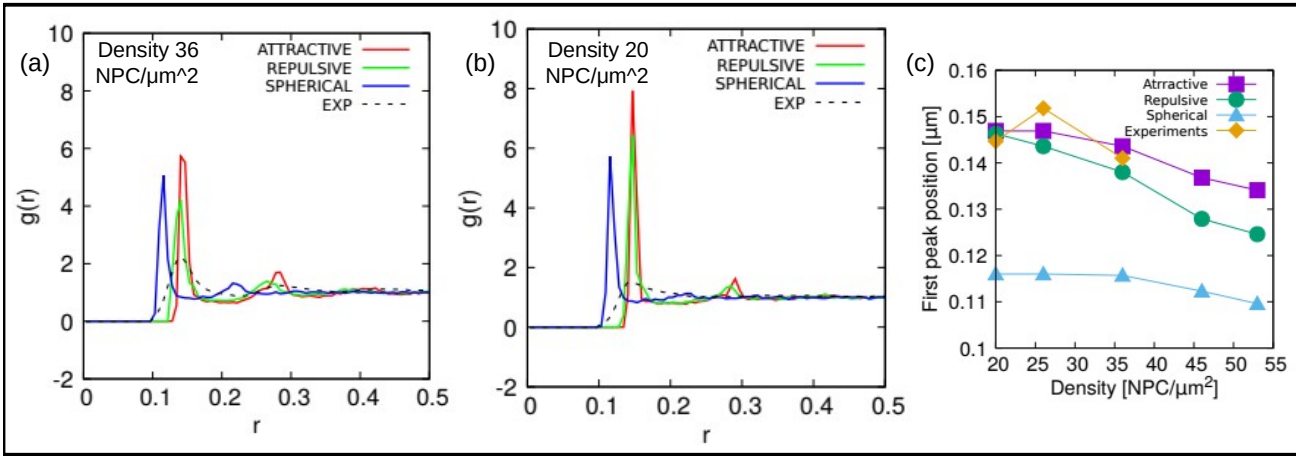

Alternative models for pore-pore interaction. (a) The radial distribution function for different interaction potential is showed: attractive octagonal potential (in red), repulsive octagonal potential (in green) and simply spherical repulsive potential (in blue). To obtain the repulsive octagonal potential we used the same LJ parameters used in the paragraph 'Model Potential for Nuclear Pores' for the attractive potential, except from the vertex-vertex interaction, for which $r_{\text {vert-vert }}^{\text {cut }}=2^{\frac{1}{6}} \sigma_{\text {vert-vert }}$. The spherical potential is a simple LJ with the following parameters: $\epsilon=5 \cdot 10^{-4} \mathrm{pg} \cdot \mu \mathrm{m}^{2} / \mu \mathrm{s}^{2}$, $\sigma=0.12 \mu \mathrm{m}, r_{c u t}=2^{\frac{1}{6}} \sigma$. All those results are obtained from simulations with density $36 \mathrm{NPC} / \mu \mathrm{m}^{2}$, and compared with the radial distribution function obtained from experimental images of Stage II oocytes (black dashed line). (b) The same results are shown for simulated densities of $20 \mathrm{NPC} / \mu \mathrm{m}^{2}$ and experimental saples on stage VI. Panel (c) shows the value of the first peak location calculated for the different effective potentials at different densities compared with the values extracted from the experimental images. 


\section{Acknowledgments}

We thank Zoe Budrikis for preliminary developments of the simulation code.

\section{References}

1. Knockenhauer KE, Schwartz TU. The nuclear pore complex as a flexible and dynamic gate. Cell. 2016;164(6):1162-1171.

2. Ris H. High-resolution field-emission scanning electron microscopy of nuclear pore complex. Scanning. 1997;19(5):368-375.

3. Wente SR, Rout MP. The nuclear pore complex and nuclear transport. Cold Spring Harbor perspectives in biology. 2010;2(10):a000562.

4. Buchwalter A, Kaneshiro JM, Hetzer MW. Coaching from the sidelines: the nuclear periphery in genome regulation. Nature Reviews Genetics.

2019;20(1):39-50.

5. Lin DH, Hoelz A. The structure of the nuclear pore complex (an update). Annual review of biochemistry. 2019;88:725-783.

6. Unwin P, Milligan R. A large particle associated with the perimeter of the nuclear pore complex. The Journal of cell biology. 1982;93(1):63-75.

7. Maul G, Deaven L, Freed J, Campbell LM, Becak W. Investigation of the determinants of nuclear pore number. Cytogenetic and Genome Research. 1980;26(2-4):175-190.

8. Fawcett DW, Chemes HE. Changes in distribution of nuclear pores during differentiation of the male germ cells. Tissue and Cell. 1979;11(1):147-162.

9. Maeshima K, Yahata K, Sasaki Y, Nakatomi R, Tachibana T, Hashikawa T, et al. Cell-cycle-dependent dynamics of nuclear pores: pore-free islands and lamins. Journal of cell science. 2006;119(21):4442-4451.

10. Sellés J, Penrad-Mobayed M, Guillaume C, Fuger A, Auvray L, Faklaris O, et al. Nuclear pore complex plasticity during developmental process as revealed by super-resolution microscopy. Scientific reports. 2017;7(1):1-8.

11. Dumont JN. Oogenesis in Xenopus laevis (Daudin). I. Stages of oocyte development in laboratory maintained animals. Journal of morphology. 1972;136(2):153-179.

12. Allan D, van der Wel C, Keim N, Caswell TA, Wieker D, Verweij R, et al.. soft-matter/trackpy: Trackpy v0.4.2; 2019. Available from:

https://doi.org/10.5281/zenodo.3492186.

13. Crocker JC, Grier DG. Methods of digital video microscopy for colloidal studies. Journal of colloid and interface science. 1996;179(1):298-310.

14. Reichelt R, Holzenburg A, Buhle E, Jarnik M, Engel A, Aebi U. Correlation between structure and mass distribution of the nuclear pore complex and of distinct pore complex components. Journal of Cell Biology. 1990;110(4):883-894.

15. Eibauer M, Pellanda M, Turgay Y, Dubrovsky A, Wild A, Medalia O. Structure and gating of the nuclear pore complex. Nature communications. 2015;6(1):1-9. 
16. Plimpton S. Fast parallel algorithms for short-range molecular dynamics. Journal of computational physics. 1995;117(1):1-19.

17. Hansen JP, McDonald IR. Theory of simple liquids: with applications to soft matter. Academic Press; 2013.

18. Jana PK, Alava MJ, Zapperi S. Irreversible transition of amorphous and polycrystalline colloidal solids under cyclic deformation. Physical Review E. 2018;98(6):062607.

19. Brüning R, St-Onge DA, Patterson S, Kob W. Glass transitions in one-, two-, three-, and four-dimensional binary Lennard-Jones systems. Journal of Physics: Condensed Matter. 2008;21(3):035117.

20. Ramasubramani V, Dice BD, Harper ES, Spellings MP, Anderson JA, Glotzer SC. freud: A Software Suite for High Throughput Analysis of Particle Simulation Data. Computer Physics Communications. 2020;254:107275. doi:https://doi.org/10.1016/j.cpc.2020.107275.

21. Yarnell J, Katz M, Wenzel RG, Koenig S. Structure factor and radial distribution function for liquid argon at $85 \mathrm{~K}$. Physical Review A. 1973;7(6):2130.

22. Ermakova E, Solca J, Huber H, Marx D. Many-body and quantum effects in the radial distribution function of liquid neon and argon. Chemical physics letters. 1995;246(3):204-208.

23. Skinner LB, Benmore C, Neuefeind JC, Parise JB. The structure of water around the compressibility minimum. The Journal of chemical physics. 2014;141(21):214507.

24. Camisasca G, Pathak H, Wikfeldt KT, Pettersson LG. Radial distribution functions of water: Models vs experiments. The Journal of chemical physics. 2019;151(4):044502.

25. Schlune A, Shahin V, Enss K, Schillers H, Oberleithner H. Plugs in nuclear pores: Transcripts in early oocyte development identified with nanotechniques. Journal of cellular biochemistry. 2006;98(3):567-576.

26. Chandler D. Introduction to modern statistical. Mechanics Oxford University Press, Oxford, UK. 1987;40.

27. Maul GG, Price JW, Lieberman MW. Formation and distribution of nuclear pore complexes in interphase. The Journal of cell biology. 1971;51(2):405-418.

28. Falcieri E, Gobbi P, Cataldi A, Zamai L, Faenza I, Vitale M. Nuclear pores in the apoptotic cell. The Histochemical Journal. 1994;26(9):754-763. 